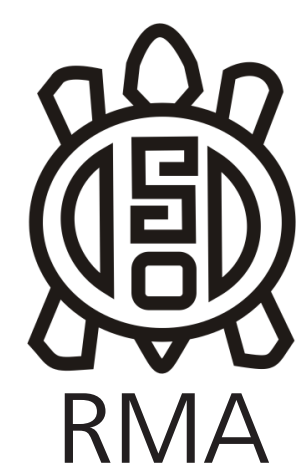

Dossier

\title{
Las palas/azadas y/o cuñas líticas de cerro El Peceño (sur de Mendoza): una primera aproximación tecnológica
}

\author{
Shovels/hoes and/or lithic wedges of El Peceño hill (southern of
}

Mendoza): a first technological approach

María Laura Salgán*, ** y María de la Paz Pompei*

\begin{abstract}
*International Center Earth Science - Malargüe, Mendoza, Argentina. E-mail: Isalgan@mendoza-conicet.gob.ar ${ }^{* *}$ CONICET, Instituto de Evolución, Ecología Histórica y Ambiente, Grupo de Estudios de Ecología Histórica y Recursos Naturales para el Desarrollo Regional-Universidad Tecnológica Nacional, San Rafael, Mendoza, Argentina.

E-mail: mpompei@mendoza-conicet.gob.ar
\end{abstract}

\begin{abstract}
Resumen
Este trabajo presenta un avance de las investigaciones llevadas a cabo en el área donde se encuentra la fuente de obsidiana El Peceño, sur de Mendoza. Se realiza una primera aproximación tecnológica de un conjunto de herramientas con morfología similar a palas/azadas y/o cuñas, confeccionadas sobre arenisca y pelita, de disponibilidad local. Se registraron $N=17$ artefactos, con una distribución espacial que coincide con las áreas de cantera-taller de obsidiana. Los estudios tecno-tipológicos muestran que los grupos humanos seleccionaron grandes lascas para manufacturar artefactos unifaciales marginales y, en menor medida, bifaciales, con morfología geométrica circular irregular. Los resultados permiten indagar acerca de su presencia en el registro arqueológico regional y, en particular, en un sitio de aprovisionamiento de rocas. La presencia de los diferentes estadios de reducción sugiere que su producción y descarte tuvo lugar en El Peceño; se propone que su función pudo estar vinculada a tareas de aprovisionamiento de obsidiana.
\end{abstract}

Palabras clave: sitio de aprovisionamiento de materias primas; herramientas de piedra; tecnología lítica; fuente El Peceño; sur de Mendoza.

\begin{abstract}
This paper presents an advance of the research carried out in the area where the obsidian source El Peceño is located, south of Mendoza (Argentina). In this work, we analyze a first technological approach of a set of lithic tools with morphology similar to shovels/hoes and/or lithic wedges, raw material available in the place. We registered $N=$ 17 artifacts worked on sandstone and pelite, whose spatial distribution coincides with the quarry-workshop areas of obsidian. Techno-typological studies show that human groups selected large flakes to manufacture marginal unifacial artifacts and, to a lesser extent, bifacial, with irregular circular geometric morphology. The results allow us to inquire about their presence in the regional archaeological record and, in particular, in a site for raw material procurement. The presence of the different stages of reduction suggests that their production and discarding took place in El Peceño; it is proposed that their function could be linked to obsidian procurement tasks.
\end{abstract}

Keywords: raw materials procurement site; stone tools; lithic technology; El Peceño source; southern of Mendoza.

\section{Introducción}

El estudio de la base regional de recursos líticos $y$, en particular, de las fuentes de aprovisionamiento de rocas, han sido en la última década los tópicos de investigación más relevantes de los estudios líticos en el sur de Mendoza (Durán et al. 2004; Gil 2006; Giesso et al. 2011; Salgán 2013; Salgán et al. 2015). En los estudios de aprovisionamiento de rocas y de la gestión de recursos líticos, las fuentes de materias primas son consideradas sitios o locus donde se encuentran evidencias de extracción y/o recolección (Carr 1994) y donde, además, se desarrollan múltiples actividades (Ericson 1984). Este marco implica el desarrollo de metodologías de muestreo que permitan dar cuenta de las actividades de extracción de rocas y de las distintas prácticas sociales involucradas en el uso de tal espacio (Colombo 2013; Bobillo \& Hocsman 2015). De este modo, en el estudio de la fuente de obsidiana ubicada en el cerro El Peceño se registraron, además de talleres y áreas de procesamiento de obsidiana, herramientas cuya morfología permite asimilarlas al tipo palas/azadas y/o cuñas. El hallazgo de estas piezas fue la apertura a interrogantes vinculados a su posible función en el sitio y/o en el registro arqueológico regional. 
Las herramientas caracterizadas como palas/azadas y/o cuñas han sido asignadas comúnmente a contextos agrícolas (Tarragó 1980; Gastaldi 2007; Pérez 2010; Escola et al. 2013); sin embargo, entre los antecedentes se mencionan usos diversos vinculados con actividades de desmalezamiento, cavado, forrajeo, recolección (Pérez 2010) y como palas y/o cuñas para actividades extractivas como la minería y el aprovisionamiento de rocas (Tripcevich et al. 2012; Colombo 2013; Figueroa et al. 2013), entre otros. En el sur de Mendoza no se cuenta con antecedentes previos de estas herramientas, asimismo el registro de plantas domésticas es aislado y su producción local aún se encuentra en discusión (Gil 2006; Gil et al. 2017). Es objetivo de este trabajo presentar una primera aproximación tecnológica de un conjunto de herramientas, novedosas para la región, y discutir sus posibles usos y funciones en el contexto de un sitio de aprovisionamiento de rocas y en el registro arqueológico regional.

\section{Antecedentes de palas/azadas y/o cuñas}

En Argentina, el abordaje de los instrumentos conocidos como palas/azadas y/o cuñas ha sido escaso y se ha dado mayoritariamente en el noroeste del país, donde su registro es abundante (e.g Boman 1992 [1908]; Von Rosen 1924; Debenedetti \& Casanova 1935; Márquez Miranda 1939; Yacobaccio 1983; Ávalos 1998; Pérez 2003, 2010; Figueroa et al. 2013). Inicialmente, la discusión se centró en la funcionalidad de dichas herramientas a partir del estudio de variables morfológicas (Pérez 2010), asociándolas a diferentes usos: actividades agrícolas (Tarragó 1980; Pérez 2010), desmalezamiento de áreas de ocupación (Medina et al. 2019), acondicionamiento y/o mantenimiento de campamentos base (Gastaldi 2007; Pérez 2010; Medina et al. 2019), excavación de tumbas (Márquez Miranda 1939; Gastaldi 2007; Pérez 2010), tareas de forrajeo y recolección (Pérez 2010), entre otros. Luego, con el advenimiento de estudios enfocados en la interpretación de las huellas de manufactura y de uso, se profundizó la discusión en torno a la forma, función y estilo de estas herramientas líticas (Yacobaccio 1983; Ávalos 1998; Gastaldi 2007; Pérez 2003, 2010, entre otros). Además, se señaló la necesidad de indagar en el aspecto tecnológico involucrado en la manufactura de las mismas (Pérez 2010) y de separar los componentes materiales, funcionales y sociales de su tecnología, con el fin de conocer la complejidad de las dimensiones sociales de la práctica tecnológica (Gastaldi 2007). Respecto al uso de las palas/azadas y/o cuñas en las fuentes de aprovisionamiento, distintos autores aluden al uso de palas para la excavación de pozos para la extracción de rocas en subsuelo, y en la minería (Tripcevich et al. 2012; Colombo 2013; Figueroa et al. 2013) la extracción de rocas por acuñamiento o palanqueo en grietas, rajaduras naturales o intencionales (Stocker \& Cobean 1984; Bloxam $\&$ Heldal 2008; Colombo 2013). Sin embargo, en general, los antecedentes coinciden en que no es común hallarlas en los contextos superficiales asociados a canteras.

En el sur de Mendoza, el incremento poblacional de los últimos ca. 2000 años AP, coincide con el registro de plantas domésticas en las poblaciones del valle del río Atuel (Gil et al. 2017) y con los primeros indicios de uso de obsidiana procedente de El Peceño (Giesso et al. 2011), la cual alcanza mayores frecuencias y distancias de transporte en los últimos ca. 600 años AP (Salgán \& Pompei 2017). En el estudio y relevamiento de otras fuentes de rocas conocidas para la región, no se han documentado hasta el momento percutores o herramientas vinculadas a las tareas de extracción y procesamiento de las materias primas. Sin embargo, en los campamentos base o sitios de actividades múltiples, se cuenta con antecedentes de guijarros de roca destinados a la formatización de herramientas (Gil 2006; Neme 2007; Salgán 2013; Pompei 2019).

\section{Cerro El Peceño: una fuente de obsidiana extra- cordillerana}

El cerro El Peceño se encuentra ubicado en la planicie oriental extra-cordillerana, a unos $5 \mathrm{~km}$ al sudeste del río Atuel (San Rafael, Mendoza) (Figura 1). Posee una altura de 1707 msnm y cubre un área aproximada de 4,5 $\mathrm{km}^{2}$ (Figura 1D). Se encuentra en la transición entre las provincias fitogeográficas de Monte y Patagonia (Cabrera 1971). Presenta cárcavas y cursos temporarios de agua que transportan material hacia bañados estacionales en un área de aproximadamente $40 \mathrm{~km}^{2}$ (Salgán \& Pompei 2017). El cerro El Peceño presenta rocas ígneas que corresponden a asomos de la Formación Cortaderas del Mioceno superior (tales como volcanitas andesíticas, dacíticas y riolíticas) y de rocas sedimentarias de la Formación El Imperial del Carbonífero superior-Pérmico inferior, entre las que se encuentran las pelitas y areniscas (Figura 1B y C) (Sepúlveda et al. 2007; Henry et al. 2014). En el cerro El Peceño es una de las seis fuentes de obsidiana conocidas para la provincia de Mendoza (Figura 1A) (Giesso et al. 2011; Salgán \& Pompei 2017).

\section{Materiales y métodos}

Se analizaron $\mathrm{N}=17$ artefactos líticos recuperados en los estudios superficiales, diseñados para el abordaje de las fuentes de aprovisionamiento del sur de Mendoza (Salgán 2013; Salgán et al. 2015; Salgán \& Pompei 2017). El muestreo superficial consistió en ocho transectas lineales y ocho cuadrículas superficiales de recolección de 0,50 $\mathrm{x}$ $0,50 \mathrm{~m}$ (cinco unidades de muestreo en el oeste y dos en la ladera noroeste) (Figura 1D). En los muestreos se recolectó la totalidad del material arqueológico observado. También se realizaron recolecciones asistemáticas de artefactos que se encontraban fuera de los muestreos; en estos casos se tomó la ubicación por medio de GPS. Una explicación detallada de la metodología llevada a cabo en El Peceño se encuentra en Salgán \& Pompei (2017).

El análisis tecnológico se realizó siguiendo los lineamientos propuestos por Aschero $(1975,1983)$ y de Aschero y Hocsman (2004). Se consideraron en particular las variables: materia prima; clase tipológica (Artefactos formatizados: unifaciales y bifaciales; núcleos, desechos 
Figura 1. Área de estudio y ubicación de cerro El Peceño. Referencias: A: Fuentes de obsidiana conocidas para la región y, en círculos negros, se indican los sitios arqueológicos conocidos: B: vista de afloramiento de arenisca; $C$ : vista de material superficial en la fuente; D: distribución superficial de los conjuntos analizados en cerro El Peceño.

Figure 1. Study area and location of El Peceño hill. References: A: Obsidian sources known to the region and in black circles, known archaeological sites are indicated; B: View of sandstone outcrop; $C$ : View of surface material at the source; D: Surface distribution of the sets analyzed in El Peceño hill.

de talla) y nódulos naturales; clase técnica; estado (entero o fracturado); porcentaje de corteza respecto al tamaño de la pieza (0\%, $50 \%, 75 \%$ y $100 \%)$; forma base; forma general del cuerpo; tamaño. El tamaño de cada pieza fue registrado de forma relativa y con calibre digital para el cálculo de volumen (medidas máximas de largo, ancho y espesor en milímetros); luego, cada valor en $\mathrm{mm}^{3}$ fue pasado a $\mathrm{cm}^{3}$ (Tabla 1). Asimismo, para poder definir las etapas de reducción presentes se tuvo en cuenta lo propuesto en Salgán (2013), donde se agrupan los tipos de desechos de talla para considerar la presencia/ausencia de las diferentes etapas de reducción.

\section{Resultados}

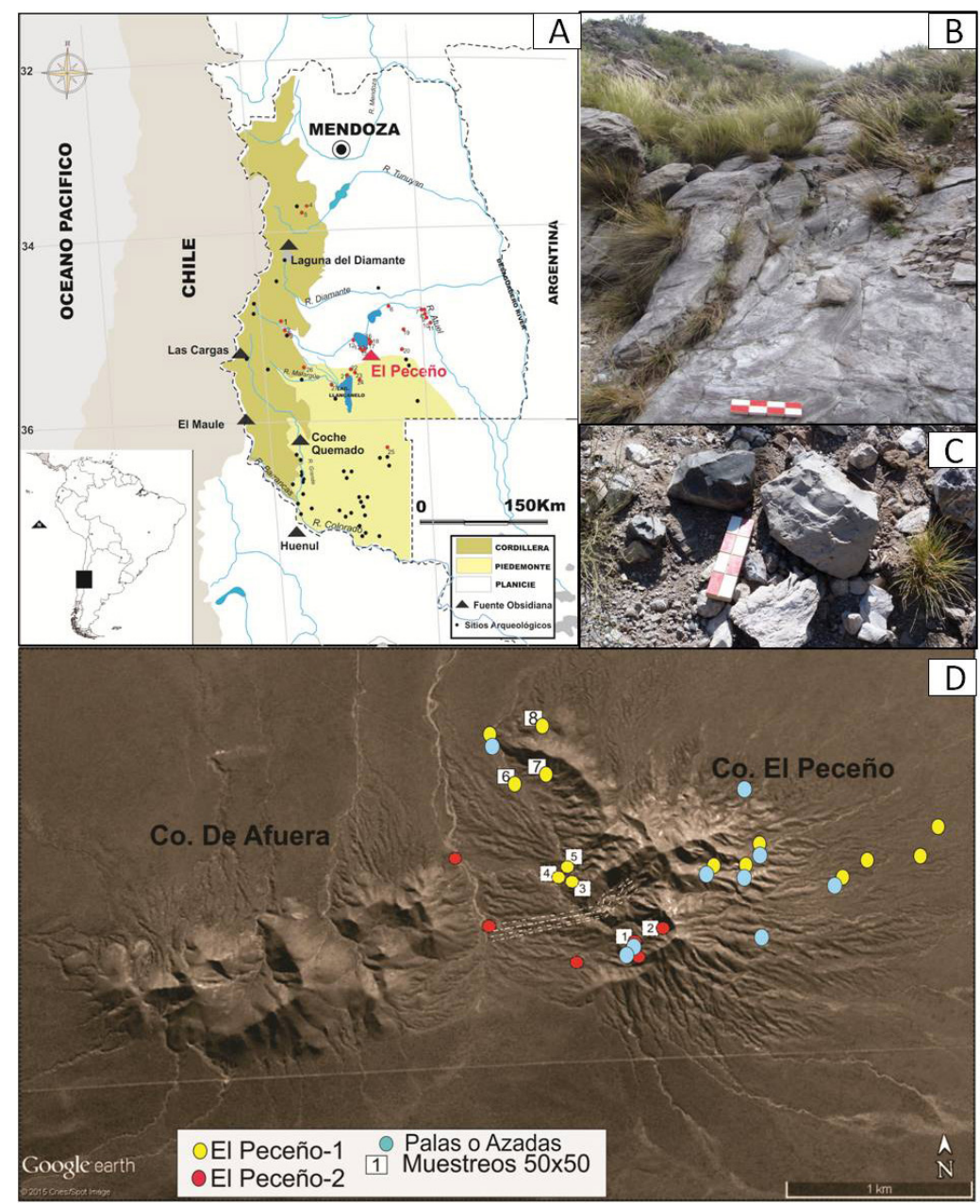

Los resultados del análisis de las palas/ azadas y/o cuñas permiten caracterizar su materia prima como arenisca $(n=15,88 \%)$ y pelita $(n=2,12 \%)$, ambas rocas de disponibilidad local en el cerro El Peceño. En la Figura 1D se observa la distribución espacial de los artefactos: el mayor número de piezas fue recuperado en el flanco este del cerro $(n=11)$, en las transectas lineales de muestreo. En el flanco oeste se documentaron seis artefactos que corresponden a la cuadrícula de muestreo 1 y a recolecciones superficiales asistemáticas.

En la Tabla 1 se observan las clases tipológicas diferenciadas: el $53 \%$ corresponde a artefactos formatizados, el $41 \%$ a desechos de talla y el $6 \%$ a núcleos. Los artefactos se encuentran en su mayoría enteros (94\%) con excepción de un artefacto con formatización unifacial trabajado sobre arenisca, que se encuentra fracturado. Los artefactos formatizados están trabajados en su mayoría sobre areniscas (88\%) y presentan color beige y rojizo, seguido por un artefacto sobre pelita (Figura 2A y B). En el 67\% de los casos, la formatización es unifacial $(n=6)$ y en el $33 \%$ $(n=3)$ bifacial. La forma base en general es lasca (88\%, $\mathrm{n}=8) \mathrm{y}$, en un caso, nodular $(12 \%, \mathrm{n}=1)$. La clase técnica o inversión de trabajo en los artefactos formatizados es baja, ya que presentan un trabajo marginal sobre la forma base lasca (Tabla 1), donde se busca una morfología de filo particular, basada en la extracción de lascas que afectan más los bordes que las caras de las piezas, en función de requerimientos en cuanto a contorno y/o bisel (sensu Aschero \& Hocsman 2004).

Las formas geométricas frecuentes de los artefactos formatizados son circular irregular (77\%) y triangular irregular (11\%), y un artefacto de formatización bifacial no diferenciado por fractura. Respecto al espesor del filo, los artefactos unifaciales presentan en promedio un espesor menor ( $x$ : 11,3 mm) a las piezas bifaciales (x: 18). El tamaño relativo (sensu Aschero1975, 1983) de los artefactos bifaciales es grandísimo (grande $=1$ y grandísimo $=2$ ), mientras que en los unifaciales es muy grande (grande=2; muy grande=2 y grandísimo=2) Al calcular el volumen en $\mathrm{cm}^{3}\left(L^{*} A^{*} E\right)$, se observa que los artefactos unifaciales ( $x$ : 409,9$)$ son, en promedio, mayores que los bifaciales ( $x$ : 362,47$)$; si bien la muestra es pequeña, las diferencias no son significativas (MannWhitney $U, p=0,897$ ) (Figura 2B). La variable espesor, en los artefactos unifaciales ( $x$ : 42,6$)$, duplica al valor medio de los bifaciales ( $x: 20)$ y corresponde a la principal diferencia de tamaño de las piezas.

Los desechos de talla, según el origen de las extracciones, pueden agruparse en lascas externas (lascas primarias, secundarias y de flanco de núcleo; 57\%) e internas 


\begin{tabular}{|c|c|c|c|c|c|}
\hline Clase Tipológica & Clase Técnica & Materia Prima & $\begin{array}{l}\text { Volumen } \\
\text { cm3 }\end{array}$ & Estado & $\begin{array}{l}\text { Corteza } \\
\%\end{array}$ \\
\hline \multirow{6}{*}{$\begin{array}{l}\text { DESECHOS DE } \\
\text { TALLA }(n=7)\end{array}$} & Lasca Angular & Arenisca & 56.37 & \multirow{2}{*}{ Entero } & $0 \%$ \\
\hline & Lasca Angular & Arenisca & 23.79 & & $0 \%$ \\
\hline & $\begin{array}{l}\text { Lasca de flanco de } \\
\text { núcleo }\end{array}$ & Arenisca & 33.12 & \multirow{2}{*}{ Entero } & $25 \%$ \\
\hline & $\begin{array}{l}\text { Lasca de flanco de } \\
\text { núcleo }\end{array}$ & Arenisca & 9.69 & & $25 \%$ \\
\hline & Lasca Secundaria & Arenisca & 15.1 & Entero & $75 \%$ \\
\hline & Lasca Primaria & Arenisca & 12.68 & Entero & $100 \%$ \\
\hline NÚCLEO $(n=1)$ & Con lascados aislados & Pelita & 111.6 & Entero & $0 \%$ \\
\hline \multirow{6}{*}{$\begin{array}{c}\text { ARTEFACTOS } \\
\text { FORMATIZADOS } \\
\text { UNIFACIALES }(n=6)\end{array}$} & $\begin{array}{l}\text { marginales, filo } \\
\text { extendido }\end{array}$ & Arenisca & 149.75 & Entero & $25 \%$ \\
\hline & $\begin{array}{l}\text { marginales, filo } \\
\text { perimetral }\end{array}$ & Arenisca & 1026.5 & Entero & $50 \%$ \\
\hline & marginal, filo extendido & Arenisca & 479.97 & Entero & $25 \%$ \\
\hline & $\begin{array}{l}\text { No diferenciado por } \\
\text { fractura }\end{array}$ & Arenisca & 38.65 & Fracturado & $0 \%$ \\
\hline & marginal, filo extendido & Arenisca & 526.91 & Entero & $25 \%$ \\
\hline & marginal, filo extendido & Pelita & 237.89 & Entero & $0 \%$ \\
\hline \multirow{3}{*}{$\begin{array}{c}\text { ARTEFACTOS } \\
\text { FORMATIZADOS } \\
\text { BIFACIALES }(n=3)\end{array}$} & $\begin{array}{l}\text { marginal, filo extendido } \\
\text { y muescas retocadas }\end{array}$ & Arenisca & 235.55 & Entero & $0 \%$ \\
\hline & marginal, filo perimetral & Arenisca & 226.99 & Entero & $25 \%$ \\
\hline & marginal, filo extendido & Arenisca & 624.85 & Entero & $0 \%$ \\
\hline
\end{tabular}

Tabla 1. Caracterización tecnológica del conjunto analizado.

Table 1. Technological characterization of the analyzed set.

El registro de palas/azadas y/o cuñas no cuenta con antecedentes en los contextos arqueológicos del sur de Mendoza y, en general, es poco usual su registro en el contexto de fuentes de aprovisionamiento de materias primas líticas (Colombo 2013). Asimismo, el uso de rocas de tipo arenisca y pelita en los conjuntos arqueológicos tampoco es frecuente, aunque no puede descartarse su presencia. En relación con los antecedentes analizados, podemos plantear que la confección de palas/azadas y/o cuñas pudo responder, al menos, a dos contextos de uso y función diferenciales. En primer lugar, se propone que estas herramientas fueron destinadas a su utilización como palas e implementadas en otros locus diferentes a la fuente, realizando su

(lascas angulares; 43\%). También se recuperó un núcleo de pelita, mediano-grande con lascados aislados y no agotado. El registro de todas las etapas de la secuencia de reducción y la presencia de reserva de corteza en el 50\% de los artefactos formatizados, nos permiten proponer que en el cerro El Peceño se presenta el total de las etapas de reducción, la cual implicaba cierta expeditividad o baja inversión en su manufactura.

\section{Discusión}

El cerro El Peceño presenta una asociación de distintas materias primas disponibles, lo que sugiere la manifestación de dos trayectorias de producción en las áreas de canteras-taller: por un lado, la formatización y uso de palas/azadas y/o cuñas sobre arenisca y pelita hasta acá descriptas; y por otro lado, el aprovisionamiento y manufactura de formas-base de obsidiana para confeccionar instrumentos, y/o para ser transportadas hacia los campamentos base (Salgán \& Pompei 2017). Las palas/azadas y/o cuñas fueron confeccionadas sobre formas base lasca, de tamaños grande a grandísimo y presentan un trabajo marginal cuyo objetivo parece responder a la búsqueda de un contorno definido (circular y triangular irregular) de las herramientas. El registro de las diferentes etapas de manufactura, sumado al estado mayormente entero de los artefactos formatizados y a la escasa inversión de trabajo observado en las piezas, nos lleva a proponer que las palas/azadas y/o cuñas fueron formatizadas, utilizadas y descartadas en el cerro El Peceño. recambio y reparación en la cantera. En apoyo a esta hipótesis, Gastaldi (2007) menciona el recambio de hojas de palas rotas en las canteras donde: "Ia/el tallador/a posiblemente iba con la pala rota (pala con el mango $y$ atado a este el fragmento de hoja roto) a la cantera, para recambiarla y volverla armar en el lugar" (Gastaldi 2007: 26). Sin embargo, en el cerro El Peceño predominan las palas/azadas y/o cuñas en estado entero y sin signos de enmangue o desgaste. Esto nos sugiere evaluar el grado de formatización de estas herramientas y observar si existe algún sector con un filo pasivo, útil para la prensión. Por otro lado, no se cuenta con antecedentes previos de estas herramientas en el registro arqueológico del sur de Mendoza, lo que debilita esta hipótesis. En segundo lugar, la manufactura, uso y descarte de dichas herramientas estarían vinculados a las tareas de extracción de obsidiana. Las tareas de aprovisionamiento requieren de una serie de herramientas tales como: percutores, cuñas, palancas, masas o percutores enmangados (Colombo 2013), según la naturaleza del yacimiento. Las cuñas o palancas permiten aprovechar grietas o fisuras naturales o intencionales, que suelen dejar rastros de respaldo en sus superficies y de lascados en ambos extremos (Colombo 2013). Por otro lado, la extracción de obsidiana con atributos específicos, tales como tamaño, ausencia de grietas térmicas y mejores aptitudes de talla, requiere de tareas extractivas como el cavado de pozos (Stocker \& Cobean 1984; Tripcevich et al. 2012). En cerro El Peceño los afloramientos de obsidiana posibilitarían el uso de cuñas o palancas para la extracción de soportes con atributos específicos. Asimismo, existen cárcavas de escurrimiento, procesos de remoción en masa y 


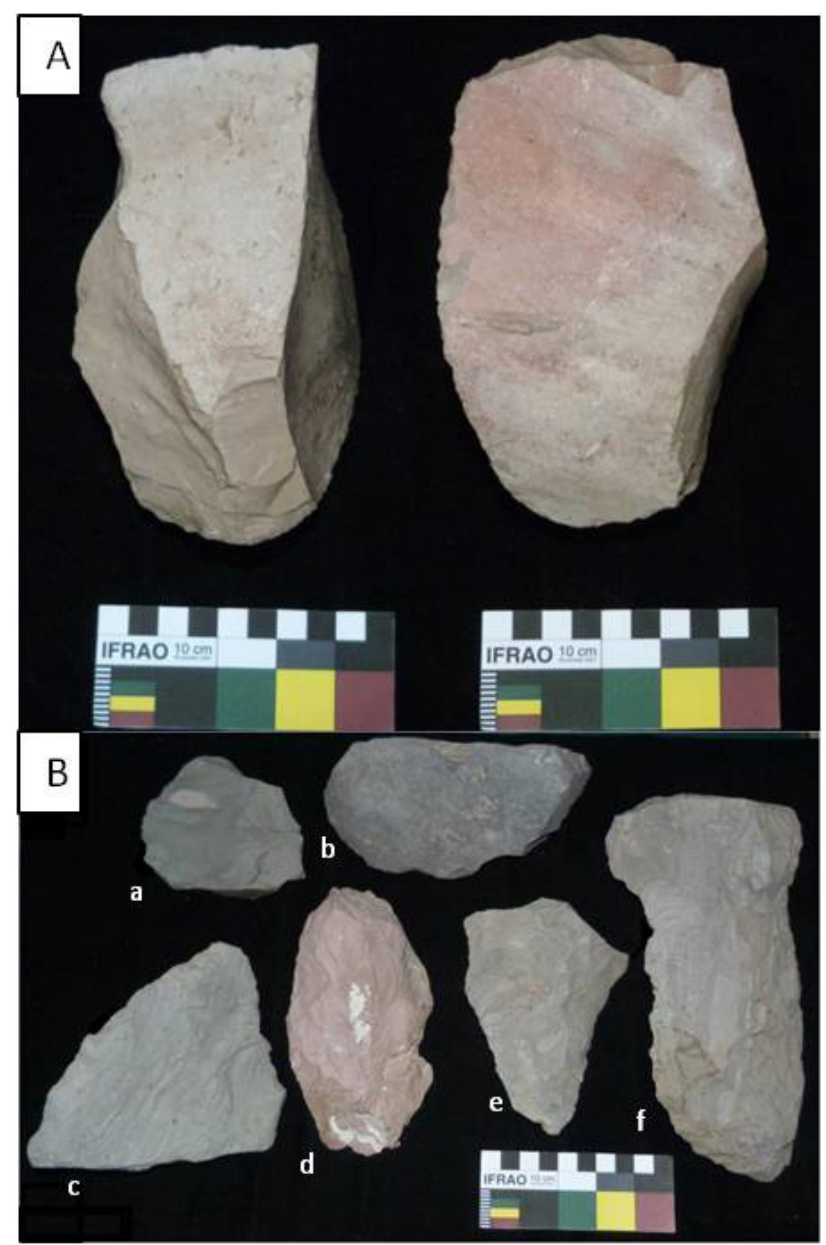

Figura 2. Palas/azadas y/o cuñas de cerro El Peceño. Referencias: A: Vista dorsal (izquierda) y ventral (derecha) de artefacto unifacial marginal trabajado sobre arenisca; $B$ : artefactos unifaciales ( $a$, b y d) y bifaciales (c, e y f) sobre arenisca.

Figure 2. Shovels / hoes and / or lithic wedges of El Peceño hill. References: A: Dorsal (left) and ventral (right) view of marginal unifacial artifact worked on sandstone; $B$ : unifacial ( $a, b$ and d) and bifacial (c, e and f) artifacts in sandstone.

enterramiento que podrían requerir el uso de palas, aunque no se han detectado hasta el momento pozos de extracción. Por lo tanto, podemos considerar que tales herramientas pudieron ser confeccionadas con el fin de extraer materias primas con atributos particulares. El análisis de las huellas de manufactura y de uso podrá ayudarnos a discernir su historia de uso y contrastar o modificar las hipótesis hasta acá propuestas.

\section{Conclusión y perspectivas}

El hallazgo de herramientas atípicas para el registro arqueológico del sur de Mendoza, nos permite proponer la realización de diferentes actividades y contextos posibles de uso que complejizan la mirada y estudio de las fuentes; al tiempo que requieren de metodologías de muestreo y análisis de abordaje interdisciplinar. En agenda, para poder contrastar las propuestas planteadas, consideramos necesario revisar las colecciones locales y regionales para descartar o documentar el uso de arenisca y pelita en los contextos de la macro región; realizar análisis funcionales como los estudios de rastros de uso (físicos y químicos); y explorar y experimentar características mecánicas de la materia prima para acciones de cavado, cuña y corte de vegetales, entre otros.

San Rafael, 15 de abril de 2019

\section{Agradecimientos}

Agradecemos a CONICET y a la ANPCyT (PICT 2014-3270), por financiar nuestra investigación. También al Dr. Walter G. Bertotto de la Universidad Nacional La Pampa por la determinación geológica de las piezas. A Gustavo Neme, Federico Bobillo y Guillermo Heider por su colaboración en los trabajos de campo. A Pedro Serra y familia, por facilitarnos la estadía. A Enrique Moreno y a los asistentes al simposio titulado "El estudio de los conjuntos líticos en las áreas de aprovisionamiento, variabilidad y formas de abordaje desde el análisis técnico-tipológico" realizado en el marco del Primer Congreso Argentino de Estudios Líticos en Arqueología (CAELA), por sus comentarios.

\section{Referencias citadas.}

Aschero, C. (1975). Ensayo para una clasificación morfológica de artefactos líticos aplicados a estudios tipológicos comparativos. (Informe a CONICET). Manuscrito no publicado, disponible en el IDEVEA-CONICET, San Rafael, Mendoza.

Aschero, C. (1983). Ensayo para una clasificación morfológica de artefactos líticos aplicada a estudios tecnológicos comparativos. Apéndice A-C. Revisión para la cátedra de Ergología y Tecnología (FFyL, UBA). Manuscrito no publicado, disponible en el IDEVEACONICET, San Rafael, Mendoza.

Aschero, C. \& Hocsman, S. (2004). Revisando cuestiones tipológicas en torno a la clasificación de artefactos bifaciales. En A. Acosta, D. Loponte \& M. Ramos (Comp.). Temas de Arqueología, Análisis Lítico (pp. 7-25). Luján, Buenos Aires: Universidad Nacional de Luján.

Ávalos, J. C. (1998). Modos de uso de implementos agrícolas de la Quebrada de Humahuaca y Puna a través del análisis de huellas de desgaste. En B. Cremonte (Ed.). Los desarrollos locales y sus territorios. Arqueología del NOA y sur de Bolivia (pp. 285-303). San Salvador de Jujuy: Universidad Nacional de Jujuy.

Bobillo, F. M. \& Hocsman, S. (2015). Mucho más que solo aprovisionamiento lítico: actividades en canteras y prácticas sociales en las fuentes de Pampa Oeste, Quebrada Seca y Punta de la Peña (Antofagasta de la Sierra, Catamarca). Revista del Museo de Antropología, 8, 23-44.

Boman, E. (1992 [1908]). Antigüedades de la región andina de la República Argentina y del desierto de Atacama. Tomo II. Traducido por D. Gómez Rubio. Universidad Nacional de Jujuy, San Salvador de Jujuy.

Bloxam, E. \& Heldal, T. (2008). Identifying heritage values and character-defining elements of ancient quarry landscapes in the Eastern Mediterranean: An integrated analysis. UE: Quarry Scapes. Conservation of Ancient Stone Quarry Landscapes in the Eastern Mediterranean, 8(10). 
Cabrera A.L. (1971). Fitogeografía de la República Argentina. Boletín de la Sociedad Argentina de Botánica, XIV, 1-42.

Carr, P. (1994). The organization of technology: impact and potential. En P. Carr (Ed.). The Organization of North American Prehistoric Chipped Stone Toll Technologies (pp. 1-8). Ann Arbor, Michigan: International Monographs in Prehistory, Archaeological Series 7.

Colombo, M. (2013). Los cazadores recolectores pampeanos y sus rocas. La obtención de materias primas líticas vista desde las canteras arqueológicas del centro de Tandilia (Tesis doctoral no publicada). Facultad de Ciencias Naturales y Museo, Universidad Nacional de La Plata, La Plata.

Debenedetti, S. \& Casanova, E. (1935). Titiconte. Publicaciones del Museo Antropológico y Etnográfico de la Facultad de Filosofía y Letras, serie A III, pp. 7-35. Buenos Aires.

Durán, V., Giesso, M., Glascock, M., Neme, G., Gil, A. \& Sanhueza, L. (2004). Estudio de fuentes de aprovisionamiento y redes de distribución de obsidiana durante el Holoceno tardío en el sur de Mendoza (Argentina). Estudios Atacameños, 28, 25-43.

Ericson, J.E. (1984). Toward the analysis of lithic production systems. En J.E. Ericson, \& B.A. Purdy (Eds.). Prehistoric quarries and lithic production (pp. 1-9). Cambridge: Cambridge University Press.

Escola, P., Hocsman, S. \& Babot, M.P. (2013). Entre las residencias y los campos de cultivo. Aportes de los cuchillos/raederas de módulo grandísimo a la cuestión del laboreo agrícola en Antofagasta de la Sierra (Puna de Catamarca) durante el primer milenio d.C. Relaciones de la Sociedad Argentina de Antropología, 38(1), 83-110.

Figueroa V., Salazar, D., Salinas, H., Núñez-Regueiro, P. \& Manríquez, G. (2013). Ergología minera prehispánica del Norte de Chile: una perspectiva arqueológica. Chungara, Revista de Antropología Chilena, 45(1), 61-81.

Gastaldi, M.R. (2007). Tecnología y Sociedad: Biografía e Historia Social de las Palas del Oasis de Tebenquiche Chico, Puna de Atacama, Primer Milenio d.C. Inglaterra: BAR International Series 1670

Giesso, M., Durán, V., Neme, G.A., Glascock, M.D., Cortegoso, V., Gil, A.F. \& L. Sanhueza. (2011). A study of obsidian source usage in the central Andes of Argentina and Chile. Archaeometry, 53(1), 1-21.

Gil, A. (2006). Arqueología de La Payunia. Inglaterra: BAR International Series 1477.

Gil, A., Menéndez, L.P., Atencio, J.P., Peralta, E.A., Neme, G. \& Ugan, A. (2017). Estrategias humanas, estabilidad y cambio en la frontera agrícola sur americana. Latin American Antiquity, 29(1), 6-26

Henry, L., Isbell, J. \& Limarino, C. (2014). The late Paleozoic El Imperial Formation, western Argentina: Glacial to post-glacial transition and stratigraphic correlations with arc-related basins in southwestern Gondwana. Gondwana Research, 25, 1380-1395.

Márquez Miranda, F. (1939). El "pucará" del pie de la cuesta de Colanzulí. Notas preliminares del Museo de La Plata II (pp. 259-269). La Plata: Museo de La Plata.

Medina, M.E., Balena, I., Vázquez, E., Coriale, N \& Pastor, S. (2019). Bosques, claros y cultivos: una aproximación tecnológicofuncional a las hachas o azuelas líticas de las Sierras de Córdoba (Argentina). Latin American Antiquity, 30(1), 142-157.

Neme, G. (2007). Cazadores-recolectores de altura en los andes meridionales: el Alto Valle del Atuel (Mendoza, Argentina). Inglaterra: BAR Internacional Series 1591.

Pérez, S. (2003). Experimentación de uso con palas y/o azadas líticas. Intersecciones en Antropología, 5, 105-117.

Pérez, S. (2010). Variabilidad en la producción de palas y/o azadas líticas de la Puna argentina. Estudios Atacameños, 40, 5-22.

Pompei, M.P. (2019). Organización de la tecnología lítica en la cuenca del río Atuel (sur de la provincia de Mendoza) durante el Holoceno tardío (Tesis doctoral no publicada). Facultad de Ciencias Naturales y Museo, Universidad Nacional de La Plata, La Plata.

Salgán, M.L. (2013). Organización tecnológica y biogeografía humana en La Payunia, sur de la Provincia de Mendoza (Tesis Doctoral no publicada). Facultad de Ciencias Naturales y Museo, Universidad Nacional de La Plata, La Plata.

Salgán, M. L., Garvey, R., Neme, G., Gil, A., Giesso, M., Glascock, M. \& Durán, V. (2015). Las Cargas: Characterization of a Southern Andean Obsidian Source and Its Prehistoric Use. Geoarchaeology. An International Journal, 30(2), 139-150.

Salgán, M.L. \& Pompei, M.P. (2017). Fuente de obsidiana El Peceño: primeros resultados de su abordaje tecnológico, geoquímico y espacial. Revista del Museo de Antropología, Suplemento Especial, 1, 51-58.

Sepúlveda, E., Bermúdez, A., Bordonaro, O. \& Delpino, D. (2007). Hoja Geológica 3569-IV, Embalse El Nihuil, provincia de Mendoza. Instituto de Geología y recursos minerales, Servicio Geológico Minero Argentino, Boletín 268, 1-52.

Stocker, T.L. \& Cobean, R.H. (1984). Preliminary report on the obsidian mines at Pico de Orizaba, Veracruz. En J.E. Ericson, \& B.A. Purdy (Eds.). Prehistoric quarries and lithic production (pp. 83-95). Cambridge: Cambridge University Press.

Tarragó, M.N. (1980). Los asentamientos aldeanos tempranos en el sector septentrional del Valle Calchaquí, Pcia. de Salta y el desarrollo agrícola posterior. Estudios Arqueológicos, 5, 29-53.

Tripcevich, N., Eerkens, J. \& Carpenter, T. (2012). Obsidian hydration at high elevation: Archaic quarrying at Chivay source, southern Peru. Journal of Archaeological Science, 39, 13601367.

Von Rosen, E. (1924). Popular account of archaeological research during the Swedish Chaco - Cordillera-Expedition, 1901-1902. Editorial Bonier, Stockholm.

Yacobaccio, H. (1983). Estudio funcional de azadas líticas del NOA. Arqueología Contemporánea, 1(1), 3-19. 